\title{
Flipping the switch for selective GSK3 inhibition
}

Glycogen synthase kinase 3 (GSK3) is a potential therapeutic target in many diseases, including cancer and neurological disorders. But clinical trials of dual GSK3 $\alpha / \beta$ inhibitors have not proved successful, and there is concern about on-target liabilities related to $\beta$-catenin signalling. Now, Wagner, Stegmaier and colleagues report the rational design of paralogue-selective GSK3 inhibitors, including a GSK3 $a$ inhibitor that suppresses leukaemia initiation and extends survival in mouse models of acute myeloid leukaemia (AML) without causing unwanted side effects.

Gene duplication events during evolution have produced multiple paralogues of several genes, such as GSK3, which exists as GSK3A on chromosome 19 and as GSK3B on chromosome 3 . The encoded enzymes are thought to have overlapping but distinct biological functions.

One concern for the potential clinical application of dual GSK3 $\alpha / \beta$ inhibitors is that such compounds can lead to activation of $\beta$-catenin, which is known to promote oncogenesis. The authors of the current study had previously found that selective genetic suppression of GSK3A impaired disease progression in AML mice without affecting $\beta$-catenin, suggesting that selective targeting of a single GSK3 paralogue might provide a route to clinical benefit.

The ATP-binding domains of GSK $3 \alpha$ and GSK3 $\beta$ differ by a single amino acid - a Glu to Asp 'switch' - in the hinge domain. Comparison of X-ray crystal structures of human GSK $3 \beta$ and a fungal GSK3 orthologue showed that the single amino acid difference in the ATP-binding domain alters hydrogen bond networks between enzyme domains, resulting in structural and topological differences.

To design a GSK3 paralogueselective inhibitor, the authors used their previously described dual GSK3 $\alpha / \beta$ inhibitor, BRD0209 (a tricyclic pyrazolotetrahydroquinolinone), as a starting point. $\mathrm{X}$-ray crystal structures of GSK3 $\beta$ in complex with BRD0209 revealed a crucial binding motif within the ATPbinding domain. Retaining the core scaffold, the team probed the effects of substituting different moieties into the pyrazole ring. This rational design approach yielded BRD0705, which showed 8-fold selectivity for GSK3a, and BRD3731, which displayed 14-fold selectivity for GSK3 $\beta$.

In HEK 293 cells expressing luciferase fusion constructs of GSK3 $\alpha$ or GSK3 $\beta$, bioluminescence resonance energy transfer readouts confirmed a $>4-6$-fold selectivity of each inhibitor.

Next, the team examined the effect of the paralogue-selective inhibitors on $\beta$-catenin in three human AML cell lines and one murine primary AML model. Notably, the GSK3aselective inhibitor, BRD0705, did not increase $\beta$-catenin stabilization in any of these settings, whereas the GSK3 $\beta$-selective inhibitor, BRD3731, induced $\beta$-catenin stabilization in two of the human AML cell lines. These results suggested BRD0705 as a more promising lead candidate for further anticancer testing.

Using Tyr297 autophosphorylation as a surrogate for GSK3a enzymatic activity, the authors showed via immunoblotting that BRD0705 inhibited GSK3 $\alpha$ activity. RNA sequencing

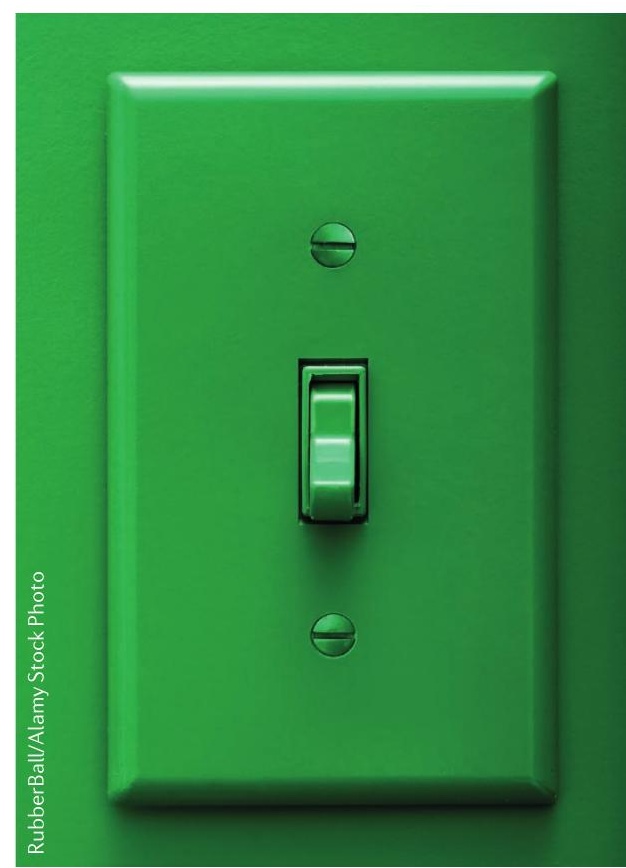

of the U937 AML cell line 24 hours after treatment with BRD0705 showed enrichment for differentiation transcription programmes and downregulation of stemness signatures.

BRD0705 impaired colony formation in six AML cell lines, as well in AML samples from five patients. Treatment had no effect on CD34 cell colony formation, providing a potential therapeutic window to selectively target leukaemic cells.

Last, in a syngeneic mouse model of AML, oral treatment with BRD0705 decreased the frequency of leukaemia-initiating cells by over threefold, and significantly inhibited development of leukaemia and improved survival. Similarly, in two orthotopic mouse models of AML, BRD0705 treatment slowed disease progression and improved survival.

Together these studies outline a rational approach to selectively target genetic paralogues and provide a lead compound to decouple the anticancer effects of GSK3a inhibition from the potentially oncogenic liabilities of dual GSK3 targeting.

Katie Kingwell

ORIGINAL ARTICLE Wagner, F. F. et al.

Exploiting an Asp-Glu "switch" in glycogen synthase kinase 3 to design paralog-selective inhibitors for use in acute myeloid leukemia. Sci. Transl Med. 10, eaam8460 (2018) 